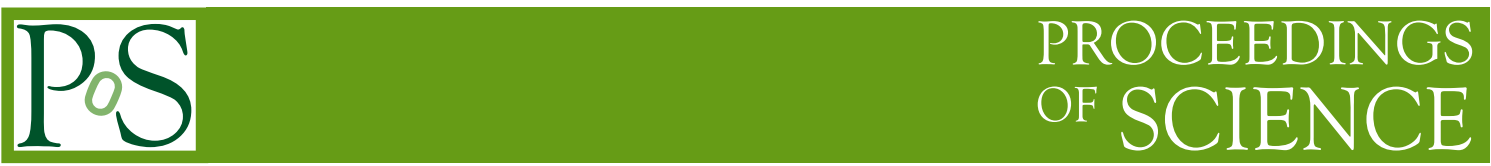

\title{
Heavy flavor collectivity in small systems
}

\section{Georgios Konstantinos Krintiras ${ }^{a, *}$}

\author{
${ }^{a}$ The University of Kansas \\ cern.ch/gkrintir \\ E-mail: gkrintir@cern.ch
}

The presence of correlations between particles significantly separated in pseudorapidity in protonproton and proton-nucleus collisions revealed surprises in the early LHC data. Are the physical processes responsible for the observed long-range pseudorapidity correlations and their azimuthal structure the same in small collision systems as in heavy ion collisions? Whereas in the case of heavy ion collisions "flow" is interpreted as generated by initial geometric inhomogeneities, calculations indicate that initial-state momentum correlations are present and could contribute to the observed azimuthal anisotropy in small systems. Probes involving heavy quarks provide us with a unique opportunity to disentangle different quantum chromodynamics effects at the boundary between low- and high- $p_{\mathrm{T}}$ interactions, and hence shed light on the origin of flow in small collision systems. A selection of the latest measurements is presented for the flow and production of heavy flavor hadrons and their decay products.

The Ninth Annual Conference on Large Hadron Collider Physics - LHCP2021

7-12 June 2021

Online

\footnotetext{
${ }^{*}$ Speaker (on behalf of the ALICE, ATLAS, and CMS Collaborations) Supported by the Nuclear Physics program DE-SC0019389 of the U.S. Department of Energy
} 


\section{Introduction}

Extensive measurements of light hadron azimuthal anisotropies (characterized by the Fourier coefficients $v_{n}$ ) have been performed in all collision systems at LHC [1], indicating the formation and hydrodynamic expansion ("flow") of a region of hot and dense quark-gluon plasma (QGP) with a small ratio of the shear viscosity to entropy density. Heavy flavor (charm and bottom) quarks have masses much larger than the typical range of temperatures in the QGP, meaning thermal production of heavy quarks during the QGP phase is suppressed relative to that of light quarks.

However, it is still postulated that charm quarks interact strongly enough to flow with the QGP. Experimental data at LHC [2-4] indeed reveal that charm quark hadrons, as well as their decay leptons, have significant azimuthal anisotropies, suggesting that they participate in the overall collective flow of the medium (see Section 2). A nonthermalized probe is required to assess the interaction with the medium more thoroughly, with the heavier bottom quark being a natural candidate. Although a series of theoretical predictions for the azimuthal anisotropies of bottom quarks exist, only limited experimental data are currently available at LHC (see Section 2).

Smaller collision systems, including proton-nucleus $(\mathrm{pPb})$ and even proton-proton ( $\mathrm{pp}$ ) collisions, have particle emission patterns with large azimuthal anisotropies that can be also described by nearly inviscid hydrodynamics. It is of interest to measure heavy flavor anisotropies in such collision systems so that we obtained information about the interaction of heavy quarks with the medium in the smallest hadronic collision systems at LHC (see Section 3). The measurement of the relative production of different heavy flavor hadron species is also sensitive to the charm- and beauty-quark fragmentation and hadron formation processes (see Section 4).

\section{Heavy flavor flow measurements in heavy ion collisions}

At LHC, a significant $v_{2}$ ("elliptic") flow signal is observed for mesons containing a charm quark, e.g., prompt $\mathrm{J} / \psi[5-7]$ and prompt $\mathrm{D}^{0}[8,9]$, while the first measurements with bottom quarks, e.g., nonprompt $\mathrm{J} / \Psi$ [6, 10], and $\mathrm{Y}(1 \mathrm{~S})[11,12]$ and $\mathrm{Y}(2 \mathrm{~S})$ [12], are compatible with zero in the kinematic region studied so far. For the charm quark case, the prompt $\mathrm{D}^{0}$ meson $v_{2}$ has been so far measured using two-particle correlation methods, while the first $v_{2}\{4\}$ measurement, i.e., using multiparticle correlations, is presented most recently [13] (Fig. 1, left). For the bottom quark case, measurements of the elliptic and "triangular" $\left(v_{3}\right)$ flow of electrons [14] (Fig. 1, right) and muons [15] originating from beauty hadron decays are also important for the understanding of the degree of thermalization of beauty quarks in the QGP. At high $p_{\mathrm{T}}$, the results can significantly discriminate between models of heavy quark energy loss and constrain heavy quark transport coefficients in the QGP, complementing measurements of the nuclear modification factor, e.g., Ref. [16].

\section{Heavy flavor flow measurements in small systems}

In small colliding systems, the study of heavy flavor hadron collectivity has the potential to disentangle possible contributions from both initial- and final-state effects. Recent observations of significant $v_{2}$ signal for prompt $\mathrm{D}^{0}[8,17]$ and prompt $\mathrm{J} / \Psi[18,19]$ in $\mathrm{pPb}$ collisions (Fig. 2, 

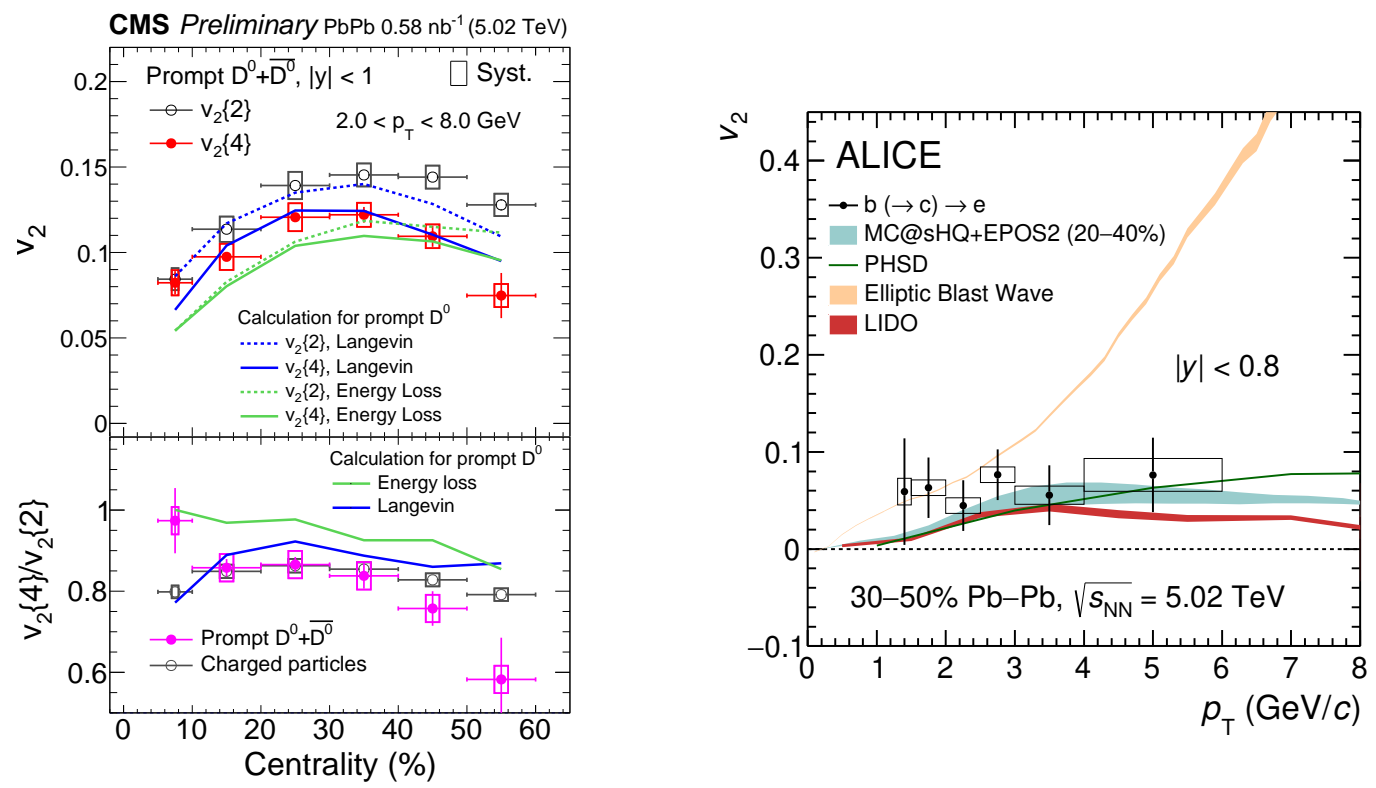

Figure 1: Left: Prompt $\mathrm{D}^{0}$ meson $v_{2}$ (upper panel) and $v_{2}$ ratios compared to those for charged particles (lower panel) as a function of the centrality class from PbPb collisions at $\sqrt{s_{\mathrm{NN}}}=5.02 \mathrm{TeV}$. The lines denote model calculations [13]. Right: Elliptic flow of electrons from bottom hadron decays in the 30-50\% centrality class in $\mathrm{PbPb}$ collisions at $\sqrt{s_{\mathrm{NN}}}=5.02 \mathrm{TeV}$ as function of $p_{\mathrm{T}}$ compared with model calculations [14].

left) provided the first evidence for charm quark collectivity in small systems. In spite of the mass differences, the observed $v_{2}$ signal for prompt $\mathrm{J} / \psi$ mesons is found to be comparable to that of prompt $\mathrm{D}^{0}$ mesons and light-flavor hadrons at a given $p_{\mathrm{T}}$ range, possibly implying the existence of initial-state correlation effects.
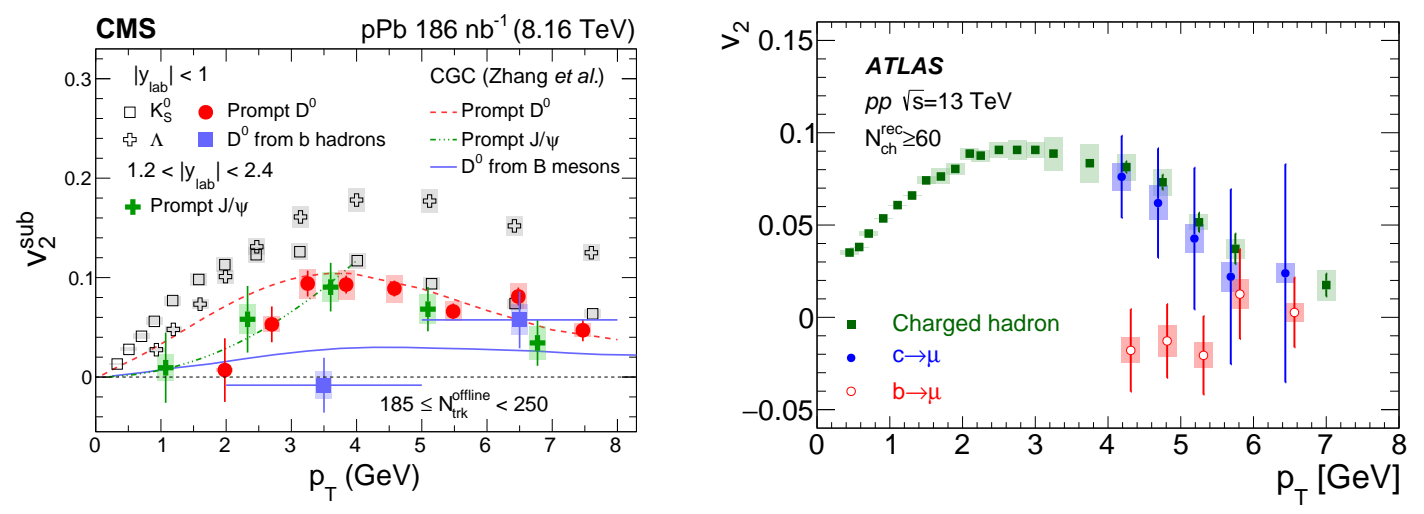

Figure 2: Left: Results of $v_{2}$ for prompt and nonprompt $\mathrm{D}^{0}$ mesons, as well as prompt $\mathrm{J} / \psi$ mesons and light flavor hadrons, as a function of $p_{\mathrm{T}}$ in $\mathrm{pPb}$ collisions at $\sqrt{s_{\mathrm{NN}}}=8.16 \mathrm{TeV}$. Lines show the theoretical calculations of prompt and nonprompt $\mathrm{D}^{0}$, and prompt $\mathrm{J} / \psi$ mesons, respectively [20]. Right: Comparison of $v_{2}$ between charged hadrons and muons originating from charm or bottom hadrons as a function of $p_{\mathrm{T}}$ in pp collisions at $13 \mathrm{TeV}$ [21]. For the case of heavy flavor muons, there are smearing effects due to decay kinematics relative to the parent hadron, so caution is warranted when comparing hadron and heavy flavor muon flow. 
Further detailed investigations start addressing open questions for understanding the origin of heavy flavor quark collectivity in small systems. These include the $p_{\mathrm{T}}$ and multiplicity dependence of charm quark collectivity in both $\mathrm{pPb}$ and pp systems (Fig. 2, right), and the details of collective behavior of beauty quarks in the $\mathrm{pPb}$ system [20]. Additionally, heavy flavor decay electrons [22] or muons [21] provide information about the anisotropies of leptons from charm and bottom hadron decays in a combined or separate way.

\section{Other measurements}

The measurement of charm and beauty hadron production is a powerful test of perturbative quantum chromodynamics calculations. The $\Lambda_{\mathrm{c}}^{+} / \mathrm{D}^{0}$ baryon-to-meson ratio in $\mathrm{pp}$ and $\mathrm{pPb}$ collisions $[23,24]$, similar to ratios measured in the light flavor sector, is larger than measurements obtained in elementary collision systems at lower center-of-mass energies, challenging the assumption that fragmentation fractions for quarks are universal. The increase of LHC Run 2 (2015-2018) data precision even allowed for the observation of a clear decreasing trend down to $p_{\mathrm{T}}=0 \mathrm{GeV}$ in the $\Lambda_{\mathrm{c}}^{+} / \mathrm{D}^{0}$ ratio for the $\mathrm{pPb}$ case [24] (Fig. 3, left). Additionally, the nuclear modification factor of $\Lambda_{\mathrm{c}}^{+}$[24] baryons is found to be higher than D mesons in the intermediate $p_{\mathrm{T}}$ region, although the current precision is not enough to draw conclusions on the role of different effects, e.g., the possible presence of hot-medium effects (Fig. 3, right).
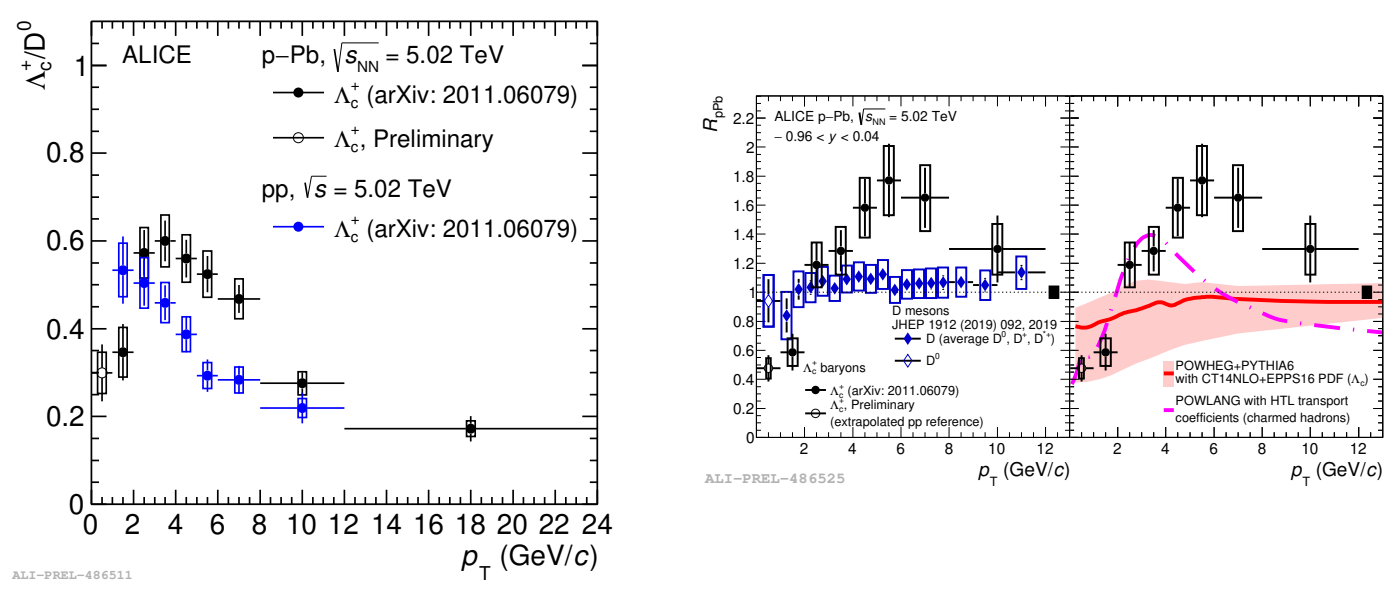

Figure 3: Left: The $\Lambda_{\mathrm{c}}^{+} / \mathrm{D}^{0}$ ratio as a function of $p_{\mathrm{T}}$ measured in $\mathrm{pPb}$ and $\mathrm{pp}$ collisions [24]. Right: The nuclear modification factor of prompt $\Lambda_{\mathrm{c}}^{+}$baryons in $\mathrm{pPb}$ collisions as a function of $p_{\mathrm{T}}$, compared to $\mathrm{D}^{0}$ mesons, as well as model calculations assuming that a hot deconfined medium is formed [24].

\section{Summary}

Heavy quarks are typically produced early in the collisions via high-momentum transfers between incoming partons. Once created, heavy quarks persist throughout the dynamical time evolution of the quark-gluon plasma, and hence serving as sensitive probes of the hot and dense medium. Although a common hydrodynamic description of azimuthal anisotropies in all colliding 
systems as resulting from initial geometry anisotropies is compelling, heavy flavor hadrons could discriminate against possible initial-state effects, e.g., gluon saturation. The increased precision of measurements with respect to those performed with the LHC Run 2 (2015-2018) data is crucial for providing further insight into heavy flavor hadron production in proton-nucleus and proton-proton collisions $[25,26]$.

\section{References}

[1] G. K. Krintiras, "Flow harmonics in heavy ion physics at CMS and ATLAS", in 55th Rencontres de Moriond on QCD and High Energy Interactions. 2021. arXiv: 2105. 07287.

[2] ALICE Collaboration, "The ALICE experiment at the CERN LHC", JINST 3 (2008) S08002, doi : 10.1088/1748-0221/3/08/S08002.

[3] ATLAS Collaboration, "The ATLAS experiment at the CERN Large Hadron Collider", JINST 3 (2008) S08003, doi : 10 . 1088/1748-0221/3/08/S08003.

[4] CMS Collaboration, "The CMS experiment at the CERN LHC", JINST 3 (2008) S08004, doi : 10.1088/1748-0221/3/08/S08004.

[5] CMS Collaboration, "Suppression and azimuthal anisotropy of prompt and nonprompt $\mathrm{J} / \psi$ production in PbPb collisions at $\sqrt{s_{\mathrm{NN}}}=2.76 \mathrm{TeV}$ ", Eur. Phys. J. C 77 (2017) 252, doi : 10.1140/epjc/s10052-017-4781-1, arXiv: 1610.00613.

[6] ATLAS Collaboration, "Prompt and nonprompt $\mathrm{J} / \psi$ elliptic flow in $\mathrm{PbPb}$ collisions at $\sqrt{s_{\mathrm{NN}}}=$ $5.02 \mathrm{TeV}$ with the ATLAS detector", Eur. Phys. J. C $\mathbf{7 8}$ (2018) 784, doi:10.1140/epjc/s10052-018-6243-9, arXiv: 1807.05198.

[7] ALICE Collaboration, "J/ $\psi$ elliptic and triangular flow in $\mathrm{PbPb}$ collisions at $\sqrt{s_{\mathrm{NN}}}=$ 5.02 TeV”, JHEP 10 (2020) 141, doi:10.1007/JHEP10 (2020)141, arXiv:2005. 14518.

[8] ALICE Collaboration, " $\mathrm{D}$ meson azimuthal anisotropy in midcentral $\mathrm{PbPb}$ collisions at $\sqrt{s_{\mathrm{NN}}}=5.02 \mathrm{TeV}$ ", Phys. Rev. Lett. 120 (2018) 102301, doi: 10.1103/PhysRevLett.120.102301, arXiv: 1707.01005.

[9] CMS Collaboration, "Measurement of prompt $\mathrm{D}^{0}$ meson azimuthal anisotropy in $\mathrm{PbPb}$ collisions at $\sqrt{s_{\mathrm{NN}}}=5.02 \mathrm{TeV}$ ", Phys. Rev. Lett. 120 (2018) 202301, doi:10.1103/PhysRevLett.120.202301, arXiv: 1708.03497.

[10] CMS Collaboration, "Suppression and azimuthal anisotropy of prompt and nonprompt J/ $\psi$ production in PbPb collisions at $\sqrt{s_{\mathrm{NN}}}=2.76 \mathrm{TeV}$ ", Eur. Phys. J. C 77 (2017) 252, doi:10.1140/epjc/s10052-017-4781-1, arXiv: 1610.00613.

[11] ALICE Collaboration, "Measurement of $\mathrm{Y}(1 \mathrm{~S})$ elliptic flow at forward rapidity in $\mathrm{PbPb}$ collisions at $\sqrt{s_{\mathrm{NN}}}=5.02 \mathrm{TeV}$ ", Phys. Rev. Lett. 123 (2019), no. 19, 192301, doi:10.1103/PhysRevLett.123.192301, arXiv: 1907.03169. 
[12] CMS Collaboration, "Measurement of the azimuthal anisotropy of $\mathrm{Y}(1 \mathrm{~S})$ and $\mathrm{Y}(2 \mathrm{~S})$ mesons in $\mathrm{PbPb}$ collisions at $\sqrt{s_{\mathrm{NN}}}=5.02 \mathrm{TeV}$ ", Phys. Lett. B 819 (2021) 136385, doi: 10.1016/j . physletb.2021.136385, arXiv:2006.07707.

[13] CMS Collaboration, "Probing charm quark dynamics via multiparticle azimuthal correlations in 5.02 TeV PbPb collisions", CMS Physics Analysis Summary CMS-PAS-HIN-20-001, 2021.

[14] ALICE Collaboration, "Elliptic flow of electrons from beauty hadron decays in $\mathrm{PbPb}$ collisions at $\sqrt{s_{\mathrm{NN}}}=5.02 \mathrm{TeV}$ ", Phys. Rev. Lett. 126 (2021) 162001, doi : 10.1103/PhysRevLett.126.162001, arXiv:2005.11130.

[15] ATLAS Collaboration, "Measurement of azimuthal anisotropy of muons from charm and bottom hadrons in $\mathrm{PbPb}$ collisions at $\sqrt{s_{\mathrm{NN}}}=5.02 \mathrm{TeV}$ with the ATLAS detector", Phys. Lett. B 807 (2020) 135595, doi:10.1016/j.physletb.2020.135595, arXiv:2003. 03565.

[16] CMS Collaboration, "Studies of beauty suppression via nonprompt $\mathrm{D}^{0}$ mesons in $\mathrm{PbPb}$ collisions at $\sqrt{s_{\mathrm{NN}}}=5.02 \mathrm{TeV}$ ", Phys. Rev. Lett. 123 (2019) 022001, doi : 10.1103/PhysRevLett.123.022001, arXiv: 1810.11102.

[17] CMS Collaboration, "Elliptic flow of charm and strange hadrons in high-multiplicity $\mathrm{pPb}$ collisions at $\sqrt{s_{\mathrm{NN}}}=8.16 \mathrm{TeV}$ ", Phys. Rev. Lett. 121 (2018) 082301, doi:10.1103/PhysRevLett.121.082301, arXiv: 1804.09767.

[18] ALICE Collaboration, "Search for collectivity with azimuthal $\mathrm{J} / \psi$-hadron correlations in high multiplicity $\mathrm{pPb}$ collisions at $\sqrt{s_{\mathrm{NN}}}=5.02$ and $8.16 \mathrm{TeV}$ ", Phys. Lett. B 780 (2018) 7, doi:10.1016/j.physletb.2018.02.039, arXiv:1709.06807.

[19] CMS Collaboration, "Observation of prompt $\mathrm{J} / \psi$ meson elliptic flow in high-multiplicity $\mathrm{pPb}$ collisions at $\sqrt{s_{\mathrm{NN}}}=8.16 \mathrm{TeV}$ ", Phys. Lett. B 791 (2019) 172, doi:10.1016/j.physletb.2019.02.018, arXiv: 1810.01473.

[20] CMS Collaboration, "Studies of charm and beauty hadron long-range correlations in pp and pPb collisions at LHC energies", Phys. Lett. B 813 (2021) 136036, doi:10.1016/j . physletb.2020.136036, arXiv:2009.07065.

[21] ATLAS Collaboration, "Measurement of azimuthal anisotropy of muons from charm and bottom hadrons in pp collisions at $\sqrt{s}=13 \mathrm{TeV}$ with the ATLAS detector", Phys. Rev. Lett. 124 (2020) 082301, doi: 10.1103/PhysRevLett.124.082301, arXiv: 1909.01650.

[22] ALICE Collaboration, "Azimuthal anisotropy of heavy flavor decay electrons in $\mathrm{pPb}$ collisions at $\sqrt{s_{\mathrm{NN}}}=5.02 \mathrm{TeV}$ ", Phys. Rev. Lett. 122 (2019) 072301, doi:10.1103/PhysRevLett.122.072301, arXiv: 1805.04367.

[23] CMS Collaboration, "Production of $\Lambda_{\mathrm{c}}^{+}$baryons in proton-proton and lead-lead collisions at $\sqrt{s_{\mathrm{NN}}}=5.02 \mathrm{TeV}$ ", Phys. Lett. B $\mathbf{8 0 3}$ (2020) 135328, doi:10.1016/j.physletb.2020.135328, arXiv: 1906.03322 . 
[24] ALICE Collaboration, " $\Lambda_{\mathrm{c}}^{+}$production in $\mathrm{pp}$ and in $\mathrm{pPb}$ collisions at $\sqrt{s_{\mathrm{NN}}}=5.02 \mathrm{TeV}$ ", arXiv:2011.06079.

[25] Z. Citron et al., "Report from Working Group 5: Future physics opportunities for high-density QCD at the LHC with heavy-ion and proton beams", CERN Yellow Rep. Monogr. 7 (2019) 1159, doi:10.23731/CYRM-2019-007.1159, arXiv: 1812.06772.

[26] ALICE Collaboration, "Future high-energy pp program with ALICE", ALICE Public Note, 2020. 